\title{
Am Vorabend der Rente mit 67 - Erkenntnis- stand und Erkenntnislücken zur Entwicklung der Erwerbschancen Älterer
}

\author{
Martin Brussig \\ Matthias Knuth
}

Die Bundesregierung hat kürzlich ihrem gesetzlichen Auftrag entsprechend einen ersten Bericht zur Lage der Älteren am Arbeitsmarkt vorgelegt, mit dem die Machbarkeit der Rente mit 67 belegt werden soll. Der Bericht betont zu Recht die in den letzten Jahren gestiegene Erwerbsbeteiligung Älterer, blendet aber derzeit schon beobachtbare Risiken beim Übergang von Erwerbstätigkeit in Rente weitgehend aus. Der vorliegende Beitrag skizziert ein komplexeres Bild von der Entwicklung der Alterserwerbstätigkeit und des Altersübergangs und benennt Forschungsfragen, die beantwortet werden müssen, um die Wirkungen der Rente mit 67 besser abschätzen zu können.

\section{Aktuelle Entwicklungen der Alterserwerbsbeteili- gung - Erwartbare Folgen der Altersgrenzenanhe- bung}

Die derzeitige Bundesregierung hält am Beschluss der Vorgängerregierung fest, beginnend mit dem Jahr 2012 die Altersgrenze für die Regelaltersrente schrittweise von 65 auf 67 Jahre anzuheben. Die Gewerkschaften kritisieren dieses Vorhaben nachdrücklich und sehen ihre Gegenargumente und Bedenken durch den aktuellen Bericht des zuständigen Bundesministeriums (vgl. BMAS 2010) ${ }^{1}$ keineswegs ausgeräumt. Es bleibt umstritten, ob und von welchen Gruppen die Lebensjahre, um die der Renteneintritt aufgeschoben wird, mit Erwerbstätigkeit ausgefüllt werden können. Nur dann kann der Aufbau der Alterssicherung für die Nacherwerbsphase auf einem akzeptablen Niveau erfolgen und die Relation zwischen Beitragszahlern und Rentenempfängern verbessert werden.

Die Einschätzungen zur künftigen Erwerbstätigkeit von Personen im rentennahen Alter gehen weit auseinander. Dies betrifft sowohl die individuelle Beschäftigungsfähigkeit als auch die strukturellen Beschäftigungsmöglichkeiten, also die Arbeitsnachfrage. Für die individuelle Beschäftigungsfähigkeit wird einerseits auf die gestiegene Lebenserwartung verwiesen, deren Anstieg sich zwar erwartbar fortsetzt, allerdings auch sozialstrukturell ungleich verteilt ist. Da zumindest in dem relevanten Alter um das 65. Lebensjahr herum die steigende Lebenserwartung für viele zu einem Gewinn an „gesunden Jahren“ führt, erscheint eine Verlängerung des Erwerbslebens zunächst realistisch (Börsch-Supan 2010). Dieser Sichtweise aber stehen Untersuchungen zu Arbeitsbelastungen gegenüber, die belegen, dass sich Arbeitsbelastungen im Urteil der Beschäftigten bislang kaum verringert haben. Folgerichtig erwartet eine große Mehrheit heute nicht, bis in ein Alter von 65 oder gar 67 Jahren zumindest in der aktuellen Tätigkeit beschäftigungsfähig zu bleiben (Ebert et al. 2006; Kistler et al. 2009). Ebenso gegensätzlich sind die Einschätzungen zur Arbeitsnachfrage. Auf der einen Seite wird mit Hilfe von Arbeitsmarktprojektionen ein zusätzlicher Bedarf von 1,2 bis 3 Mio. Arbeitsplätzen ermittelt, der sich aus der längeren Erwerbstätigkeit ergibt (Fuchs 2006). Dies würde die schon heute bestehenden Beschäftigungsprobleme auch der Älteren verschärfen (Guggemos et al. 2007). Auf der anderen Seite wird gerade in regionaler Betrachtung der „Fachkräftemangel“ diskutiert (Böckler 2010; Fuchs / Zika 2010; Lutz 2010), der durch längere Erwerbsphasen zumindest vorübergehend abgemildert werden könnte.

Künftige Entwicklungen sind naturgemäß schwer zu erfassen. Mit Blick auf die
„Rente mit 67“ gibt es aber einen zentralen Anhaltspunkt, um die Auswirkungen für die Erwerbstätigkeit im Alter abzuschätzen: die zurückliegenden Rentenreformen. Denn schon in den vergangenen Jahren seit 1997 - wurden Altersgrenzen in der Gesetzlichen Rentenversicherung angehoben. Ein vorzeitiger Zugang ist derzeit zwar immer noch möglich, wird aber mit

\footnotetext{
1 Die aktuelle Berichterstattung erfolgte aufgrund der "Revisionsklausel“ des BMA zur sozialpolitischen Vertretbarkeit der Altersgrenzenanhebung: „Die Bundesregierung hat den gesetzgebenden Körperschaften vom Jahre 2010 an alle vier Jahre über die Entwicklung der Beschäftigung älterer Arbeitnehmer zu berichten und eine Einschätzung darüber abzugeben, ob die Anhebung der Regelaltersgrenze unter Berücksichtigung der Entwicklung der Arbeitsmarktlage sowie der wirtschaftlichen und sozialen Situation älterer Arbeitnehmer weiterhin vertretbar erscheint und die getroffenen gesetzlichen Regelungen bestehen bleiben können" (§ 154, Abs. 4 SGB VI).
}

Martin Brussig, Dr., wissenschaftlicher
Mitarbeiter am Institut Arbeit und
Qualifikation (IAQ) der Universität
Duisburg-Essen. Arbeitsschwerpunkte:
Arbeitsmarktsoziologie, Arbeitsmarktpolitik.
e-mail: martin.brussig@uni-due.de
Matthias Knuth, Prof. Dr., Leiter der
Abteilung "Arbeitsmarkt-Integration
- Mobilität" am IAQ der Universität
Duisburg-Essen. Arbeitsschwerpunkte:
Erwerbssoziologie, Soziologie des
Wohlfahrtsstaates.
e-mail: matthias.knuth@uni-due.de


Abschlägen vom Rentenanspruch belegt. Für die „Altersrente wegen Arbeitslosigkeit und nach Altersteilzeitarbeit" wird gegenwärtig (2006 bis 2012) das frühestmögliche Renteneintrittsalter von 60 auf 63 Jahre angehoben. Das abschlagsfreie Zugangsalter ist um fünf Jahre gestiegen, und für eine arbeitsmarktrelevante Rentenart hat sich das frühestmögliche Zugangsalter um drei Jahre erhöht, also um jeweils mehr als die zwei Jahre, um die es bei der Rente mit 67 geht. Zudem vollzog bzw. vollzieht sich die aktuelle Anhebung schneller als es bei der Rente mit 67 geplant ist, nämlich innerhalb von 15 Jahren (1997 bis 2012) statt in 21 Jahren (2012 bis 2031). Nicht zuletzt: Der aktuelle Anhebungsprozess verteilt sich auf nur elf Kohorten (1937 bis 1947), der künftige dagegen auf 18 Kohorten (1947 bis 1964). Die zurückliegenden Jahre waren also bereits stärker reformgeprägt als es für die Zukunft zu erwarten ist.

Aus den individuellen Reaktionen auf das veränderte institutionelle Umfeld, so die These des vorliegenden Beitrages, lässt sich ablesen, welche Entwicklungen bei der Anhebung der Altersgrenze für die Regelaltersrente auf 67 Jahre zu erwarten sind. Die „Rente mit 67“ wird also als eine graduelle Fortsetzung der bisherigen Reformen betrachtet. Die Übertragbarkeit der Erfahrungen aus den jüngst durchlaufenen Rentenreformen ist jedoch durch zwei Aspekte eingeschränkt: Erstens ist gegenwärtig noch ein vorzeitiger, mit Abschlägen behafteter Rentenzugang möglich; die künftige Altersgrenzenanhebung wird auch die Möglichkeit zum vorzeitigen Rentenzugang erheblich beschneiden. Diese Besonderheit der aktuellen Altersgrenzenanhebung kommt aber dem Anliegen entgegen, die „Mechanismen“ des Erwerbsaustritts und des Rentenbeginns zu analysieren, denn der Rentenbeginn lässt sich so eher als ein individueller und betrieblicher, nicht ausschließlich institutionell determinierter Entscheidungsprozess verstehen. Zweitens steht ein jeweils unterschiedliches Alter zur Debatte (60 bis 63 bzw. 65 bis 67), für das möglicherweise das individuelle Arbeitsvermögen grundsätzlich unterschiedlich zu bewerten ist. Jedoch war die gerontologische Forschung bislang nicht in der Lage, feste chronologische Altersgrenzen für eine Erwerbstätigkeit zu identifizieren; sie hat lediglich die Kontextabhängigkeit hierfür betont.

Auch der Bericht der Bundesregierung zur Altersgrenzenanhebung stützt sich auf Veränderungen in der Alterserwerbsbeteiligung in der jüngsten Vergangenheit. Allerdings geschieht dies nicht, um die Chancen und Grenzen der künftigen Entwicklung auszuloten, sondern um die Machbarkeit der Rente mit 67 zu betonen. Die Erreichbarkeit der Rente mit 67 aus Erwerbstätigkeit heraus, Probleme beim Altersübergang sowie Schwierigkeiten in der individuellen Alterssicherung werden beinahe gar nicht thematisiert. Wir argumentieren in diesem Beitrag, dass für ein robustes Verständnis der „Rente mit 67“ der Blick auf die jüngst durchlaufenen Entwicklungen durchaus aufschlussreich ist, zugleich aber nicht nur die Ursachen der steigenden Erwerbsquote, sondern auch die neuen und alten Probleme beim Altersübergang einbezogen werden müssen.

Wir beschreiben zunächst die Entwicklungen der Alterserwerbsbeteiligung im reformgeprägten Umfeld seit Mitte der 1990er Jahre (Abschnitt 2). Der anschließende Blick auf die aktuellen Entwicklungen in der Alterserwerbstätigkeit verdeutlicht alte und neue Problemfelder, die schon heute vorhanden sind und deren Schwierigkeiten sich verstärken werden, wenn der Druck auf eine weitergehende Verlängerung der Erwerbsphasen anhält (Abschnitt 3). Anhand dieser Betrachtung wie auch anhand vorhandener Wissenslücken über die $\mathrm{Zu}$ sammenhänge zwischen Alterserwerbstätigkeit und Renteneintritt zeigt sich ein Forschungsbedarf, der befriedigt werden muss, um zu einem komplexen Verständnis der Wirkungen der Altersgrenzenanhebung zu gelangen, auf das sich eine empirisch informierte Politik stützen könnte (Abschnitt 4). Abschnitt 5 zieht ein Fazit. ${ }^{2}$

\section{Methodische Anmerkun- gen: Querschnitts- und Kohortenbetrachtung}

Die zentralen Indikatoren zur Analyse der Alterserwerbstätigkeit, wie sie etwa vom Statistischen Bundesamt, der Deutschen Rentenversicherung oder von Eurostat bereitgestellt und auch von der Bundesregierung in ihrem Bericht verwendet werden, sind die Alterserwerbsquote - der Anteil der Erwerbstätigen an der Bevölkerung zwischen 55 und unter 65 Jahren -, das durchschnittliche Rentenzugangsalter und das durchschnittliche Erwerbsaustrittsal- ter. Alle drei Indikatoren beziehen sich in der Regel auf Kalenderjahre, deren Vergleich eine Einschätzung der historischen Entwicklung erlauben soll.

Diese Indikatoren hängen sachlich weniger zusammen als gemeinhin angenommen wird: Weder fallen Erwerbsaustritt und Renteneintritt notwendigerweise zeitlich zusammen, noch liegt der Erwerbsaustritt zwingend vor dem Renteneintritt. Die Alterserwerbsquote kann ansteigen, ohne dass sich das Erwerbsaustrittsalter ändert, indem etwa die Frauenerwerbstätigkeit im Alter steigt. Zunehmende Frauenerwerbstätigkeit konnte in der Vergangenheit sogar zu einem sinkenden Renteneintrittsalter führen, da für mehr Frauen die an Erwerbstätigkeit geknüpfte, vorzeitig beziehbare, „Altersrente für Frauen" zugänglich wurde. Tatsächlich entwickelten sich Alterserwerbsquote, Erwerbsaustrittsalter und das Renteneintrittsalter nicht gleich gerichtet: Während die Alterserwerbsquote in den Jahren bis ca. 2003 stagnierte und danach deutlich anstieg, ist das Rentenzugangsalter bis 2006 gestiegen und stagniert seitdem, und das durchschnittliche Erwerbsaustrittsalter stieg bis 2003, stagnierte dann und ist zuletzt sogar gesunken (Abbildung 1).

In diesem scheinbar widersprüchlichen Bild schlägt sich nicht nur nieder, dass der Erwerbsaustritt zum Teil von anderen Faktoren beeinflusst wird als der Rentenzugang. Es wirkt sich auch ein prinzipieller Mangel von Querschnittsindikatoren aus, der nur selten explizit angesprochen wird, nämlich die unterschiedliche Besetzung der Kohorten, die in die Berechnung des Durchschnittswertes für ein Kalenderjahr eingehen. Beispielsweise geht zwischen 55 und 64 Jahren die Erwerbsbeteiligung mit fortschreitendem Alter zurück. Wenn in einem Jahr die Gruppe der 55- bis unter 65-Jährigen durch geburtenstarke Jahrgänge bei den 60 - bis 64 -Jährigen und geburtenschwache Jahrgänge bei den 55 bis 59-Jährigen gekennzeichnet ist, ist die durchschnittliche Erwerbsbeteiligung der 55- bis 64-Jährigen niedriger als in einem anderen Jahr, in dem die Größe der Ge-

\footnotetext{
2 Grundlage der hier vorgestellten Ergebnisse ist der Altersübergangs-Monitor, ein internetbasiertes Berichtssystem zur Alterserwerbstätigkeit und zu Altersübergängen (http://www.iaq.uni-due.de/ auem-report/ ). Der Altersübergangs-Monitor wird seit 2003 von der Hans-Böckler-Stiftung und seit 2006 auch vom Forschungsnetzwerk Alterssicherung der Deutschen Rentenversicherung Bund gefördert.
} 
Abb. 1: Alterserwerbsquote ( 55 bis unter 65 Jahre), Rentenzugangsalter und Erwerbsaustrittsalter (2001 bis 2008)

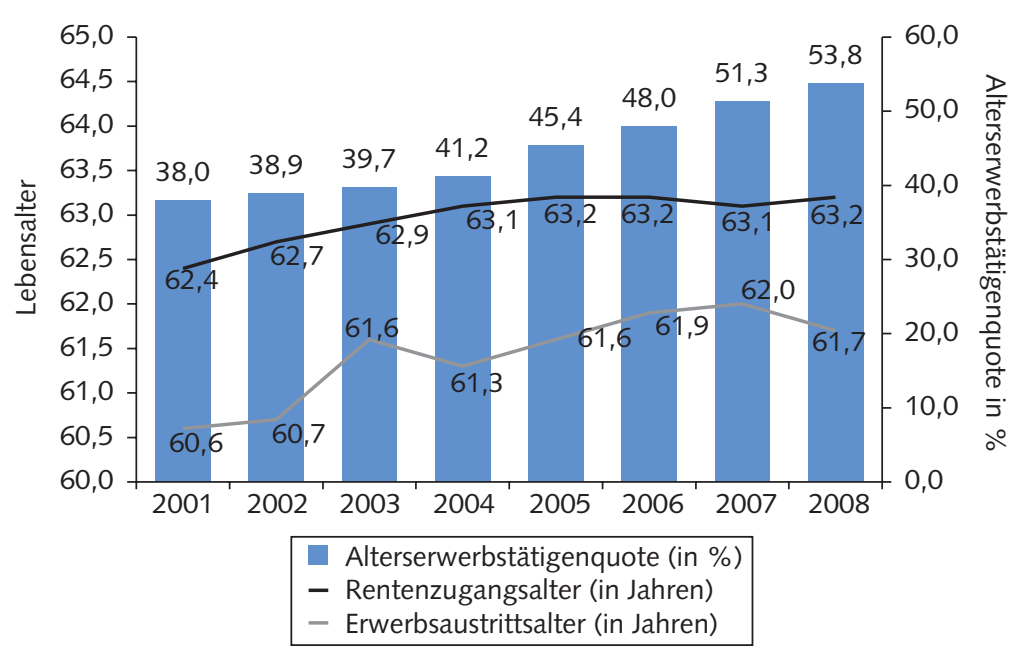

Quelle: Brussig 2010d, e, basierend auf Mikrozensus, Deutsche Rentenversicherung und Eurostat.

WSI MITTEILUNGEN

burtskohorten genau anders herum verteilt ist, auch wenn sich am Erwerbsverhalten der Individuen nichts geändert hat. Mit anderen Worten: Die durchschnittliche Alterserwerbsbeteiligung in einer Altersspanne kann sich ändern, ohne dass sich das Erwerbsverhalten ändert. Ein Blick auf die demografische Struktur der relevanten Altersjahre zwischen 2001 und 2013 zeigt, dass es erhebliche Unregelmäßigkeiten in der Besetzung einzelner Jahrgänge gerade in diesem Zeitraum gibt: Im Jahr 2001 gab es mehr Personen zwischen 60 und unter 65 Jahren als im Alter zwischen 55 und unter 60 Jahren. 2007 sind beide Altersgruppen etwa gleich groß, und 2013 werden die 55bis unter 60-Jährigen überwiegen (Abbildung 2).

Durch die „mikrodemografischen Verwerfungen“ sind die Querschnittsindikatoren nicht „falsch“, aber sie lassen sich nicht ausschließlich als Ergebnis von individuellem Handeln und individuellen Entscheidungen - und folglich auch nicht als Reaktion auf ein geändertes institutionelles Umfeld - deuten. Hierfür sind, wie wir weiter unten ausführen werden, Analysen einzelner Kohorten sehr viel geeigneter, da sich dann die Kohortenstärke nicht mehr auswirkt.

\section{Der Einstieg in die Verlän- gerung der Erwerbsphase scheint geglückt ...}

Trotz einer anhaltenden Ablehnung der Rente mit $67^{3}$ gibt es schon seit Längerem deutliche Anzeichen, dass sich viele Menschen auf eine längere Erwerbstätig-

\section{Abb. 2: Altersstruktur der Bevölkerung zwischen $\mathbf{5 0}$ und unter 70 Jahren $(2001,2007$ und 2013)}

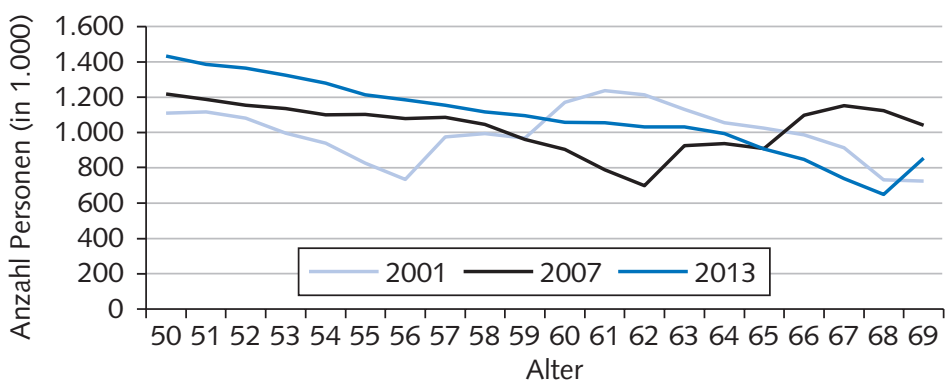

keit und einen späteren Renteneintritt einstellen. So sank unter den 40-Jährigen (und älter) der Anteil jener, die damit rechneten, mit spätestens 60 Jahren aus dem Erwerbsleben auszuscheiden, von 1996 bis 2002 von 50 auf $35 \%$. In diesem Zeitraum war allerdings keine Zunahme bei denjenigen zu beobachten, die erwarteten, später als mit 60 Jahren in den Ruhestand zu wechseln. Zugenommen hatte vor allem die Ungewissheit über das eigene Renteneintrittsalter (von 18 auf $32 \%$ ) (Engstler 2004). Im Jahr 2008 gab knapp die Hälfte der Erwerbstätigen zwischen 55 und unter 65 Jahren an, auch nach dem Renteneintritt noch erwerbstätig sein zu wollen (vgl. Dobritz/Micheel 2010), wobei in dieser Umfrage die Art und der Umfang der Beschäftigung offen geblieben sind. Doch es sind nicht nur die individuellen Planungen zu Erwerbstätigkeit und Ruhestand, die sich geändert haben, sondern auch das beobachtbare Verhalten hat sich gewandelt. Der Einstieg in die Verlängerung der Erwerbsphase scheint geglückt, wie sich anhand unterschiedlicher Indikatoren zeigen lässt.

Das durchschnittliche Rentenzugangsalter ist zwischen 1999 und 2008 um ein Jahr auf 63,2 Jahre gestiegen (Abbildung 1). Lag noch im Jahr 2000 das häufigste Zugangsalter in Altersrenten bei 60 Jahren, so lag es 2008 bei 65 Jahren; für die Jahre 2009 und 2010 ist zu erwarten, dass (bezogen auf ihren jeweiligen Anteil in der Bevölkerung) mehr 63-Jährige als 60-Jährige in Altersrente gehen. Der spätere Renteneintritt gilt also auch kohortenspezifisch und ist nicht allein durch die demografisch bedingte ungleiche Besetzung der Altersjahrgänge im Renteneintrittsalter verursacht (vgl. Brussig 2010e).

Die „mikrodemografischen Ungleichgewichte" haben aber bewirkt, dass bis 2004 der Anstieg des Rentenzugangsalters überschätzt wurde, während die seit $2007 \mathrm{zu}$ beobachtende Stagnation im Querschnitt den auf der Ebene von Geburtskohorten weiterhin zu verzeichnenden Aufschub des Rentenbeginns verdeckt. In den Jahren ab 2009 ist sogar mit einem sinkenden durchschnittlichen Renteneintrittsalter zu rechnen, weil geburtenstarke Jahrgänge potenzieller Frührentnerinnen und -rentner

3 Im Sommer 2008 bewerteten in einer repräsentativen Stichprobe der 55- bis unter 65-Jährigen in Deutschland zwei Drittel die Anhebung des Rentenalters als "schlecht" bzw. "sehr schlecht", vgl. Dobritz/Micheel 2010, S. 1. 
nachrücken. Ein sinkendes Durchschnittsalter von Rentenzugangskohorten wäre deshalb nicht zwangsläufig ein Anzeichen für einen Trendbruch in den Bemühungen, den Rentenbeginn auf spätere Lebensjahre zu verschieben. Detaillierte Analysen des Zugangsalters bei einzelnen Rentenarten zeigen, dass der Anstieg des Zugangsalters in Übereinstimmung mit den durch die Rentenabschläge gesetzten Anreizen begann (Büttner 2005). Im Bericht der Bundesregierung wird hervorgehoben, dass die Neurentnerinnen und -rentner mit 65 Jahren zu wachsenden Anteilen direkt aus versicherungspflichtiger Beschäftigung kommen (BMAS 2010, S. 18): Waren im Jahr 2000 nur $9 \%$ der Neurentnerinnen und -rentner mit 65 Jahren unmittelbar vor der Rente erwerbstätig, so waren es im Jahr 2009 schon $29 \%$. Dabei wird aber unterschlagen, dass im gleichen Zeitraum die Altersteilzeitbeschäftigung zugenommen hat und sehr viele die Altersteilzeit so terminiert haben, dass sie abschlagsfrei - also mit 65 Jahren - in Rente wechselten, dank des Blockmodells der Altersteilzeit aber schon vorher aus aktiver Beschäftigung ausschieden und dennoch - da weiterhin Lohn- bzw. Gehaltsempfänger und nicht Rentenbezieher - als erwerbstätig galten.

Die Erwerbstätigenquote der 55- bis unter 65-Jährigen ist in den letzten zehn Jahren erheblich gestiegen: von 38,0\% (2001) auf 53,8 \% im Jahr 2008 (Abbildung 1). Entsprechend dem Messkonzept von Erwerbstätigkeit sind hier alle Erwerbstätigen ohne eine Mindestgrenze - und deshalb u.a. auch erwerbstätige (Früh-)Rentner - enthalten. Bezogen auf den Ausgangswert, entspricht dies einer Zunahme von nahezu $50 \%$. Auch hier wirken sich die „mikrodemografischen Ungleichgewichte" aus. Ihr Effekt auf den Anstieg der Alterserwerbstätigenquote seit 2001 lässt sich berechnen, indem ermittelt wird, wie hoch die Alterserwerbstätigenquote gewesen wäre, wenn die altersspezifischen Erwerbstätigenquoten des Jahres 2001 auf die demografische Struktur des Jahres 2007 übertragen werden und anschließend die Erwerbsbeteiligung für die 55- bis unter 65-Jährigen ermittelt wird. Die Alterserwerbstätigenquote hätte dann bei 41,7 \% gelegen, d.h. 3,7 Prozentpunkte oder etwa $20 \%$ des Anstiegs der Alterserwerbsbeteiligung gehen auf die - für diesen Indikator - „günstigere“ Altersstruktur zurück.

Das heißt aber auch, dass der Großteil des Anstiegs der Alterserwerbstätigenquo-

\section{Abb. 3: Altersspezifische Erwerbstätigenquote}

- ausgewählte Kohorten -

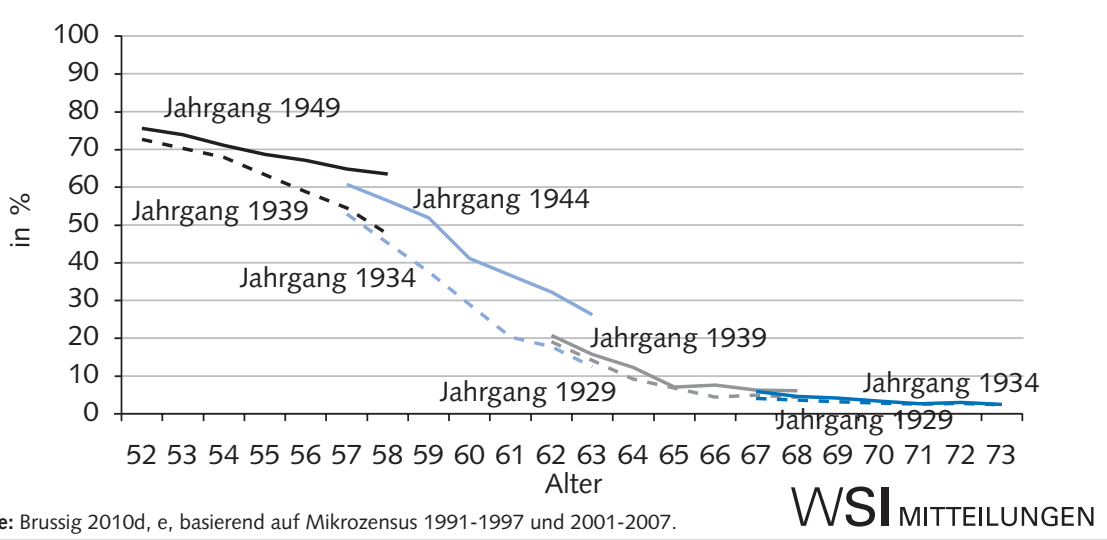

Quelle: Brussig 2010d, e, basierend auf Mikrozensus 1991-1997 und 2001-2007.

te auf ein verändertes Erwerbsverhalten zurückzuführen ist. Im Kohortenvergleich wird deutlich, dass sowohl die Alterserwerbsbeteiligung gestiegen ist - in der Kohorte der 1949 Geborenen waren im Alter von 58 Jahren fast zwei Drittel noch erwerbstätig, während es in der Kohorte der 1939 Geborenen im gleichen Alter nicht einmal die Hälfte war - als auch die Erwerbsphasen sich auch Konjunkturzyklen übergreifend verlängert haben (Abbildung 3). Der altersbedingte Rückgang der Erwerbstätigenquote vollzieht sich in den später geborenen Kohorten langsamer als unter den früheren Kohorten. Dies ist besonders auffällig für den Altersabschnitt zwischen 52 und 58 Jahren, bislang aber kaum ausgeprägt für den Abschnitt zwischen 62 und 68 Jahren. Ermittelt man die Verlängerung des Erwerbslebens nicht wie Eurostat als „durchschnittliches Erwerbsaustrittsalter pro Kalenderjahr“, sondern stattdessen wie eine Überlebensrate,${ }^{4}$ dann zeigt sich erstens, dass sich in kohortenspezifischer Betrachtung die Erwerbsphasen erheblich verlängert haben und dass dies - zweitens - etwa $50 \%$ des Anstiegs der Alterserwerbsquote zwischen 2001 und 2008 von 38,0 auf 53,8 \% (s.o.) ausmacht (vgl. Brussig 2010d). Weitere ca. $30 \%$ sind auf die steigende Frauenerwerbsbeteiligung zurückzuführen.

Trotz anhaltender Differenzen in der Alterserwerbstätigenquote vollzog sich ihr Anstieg sowohl in West- als auch in Ostdeutschland, bei Männern und Frauen sowie auf allen Qualifikationsniveaus. Die Zunahme der Alterserwerbstätigenquote ist nicht aufTeilzeitarbeit und geringfügige Beschäftigung beschränkt (obwohl beides überproportional zugenommen hat), son- dern umfasst auch vollzeitige bzw. sozialversicherungspflichtige Beschäftigungsverhältnisse.

\section{1 ... doch alte Probleme bestehen fort, neue treten hinzu}

Der Bericht der Bundesregierung zeichnet sein Bild von der Erwerbstätigkeit der Älteren auf Grundlage der Erwerbstätigenquote und des Durchschnittsalters beim Renteneintritt. Dies ist unvollständig, solange die Qualität der Altersübergänge und der Beschäftigungsverhältnisse ausgeblendet bleibt. In qualitativer Hinsicht bestehen nicht nur alte, seit Langem virulente Probleme der Alterserwerbstätigkeit fort, vielmehr sind auch neue Probleme entstanden, die zu einem guten Teil die anhaltenden Kontroversen um die „richtige“ Rentenaltersgrenze erklären.

Zu den alten, aber nach wie vor anhaltenden Problemen zählen hohe Arbeitsbelastungen und ihre Folgen für die Beschäftigungsfähigkeit. Über die individuellen Erwerbsverläufe hinweg ist eine hohe Konstanz der Arbeitsbedingungen und mithin für die Personen mit stark belastenden Arbeitsbedingungen eine lange Expositionsdauer festzustellen. $89 \%$ derjenigen, die 1995 „körperlich schwer gearbeitet“ haben, gaben dies auch 2001 an, sofern sie

4 Berechnet wird der Rückgang der Erwerbsquote innerhalb einer Kohorte bis auf einen Wert von $50 \%$, bezogen auf die Erwerbsquote, den diese Kohorte im Alter von 55 Jahren (den Beginn des Altersspektrums von 55 bis unter 65 Jahren) hatte. 
noch erwerbstätig waren. Unter denen, die 1985 körperlich schwer gearbeitet haben, waren es 2001 immer noch $82 \%$ (Werte für 1985-2001 nur für Westdeutschland) (Trischler/Kistler 2010, S. 37). Soweit man das auf Grundlage subjektiver Einschätzungen sagen kann - für die sich die Maßstäbe im Zeitverlauf geändert haben mögen -, haben sich die wahrgenommenen körperlichen Arbeitsbedingungen in einem Zeitraum von ca. 20 Jahren (1985-2006) kaum geändert (ebd., S. 22), während die psychischen Belastungen gestiegen sind.

Hohe Arbeitsbelastungen erschweren die Erwerbstätigkeit bis zum Rentenalter massiv und führen zu vorzeitigen Erwerbsaustritten. Am deutlichsten drückt sich dies im Zugang zu Erwerbsminderungsrenten aus. Es gibt einen klaren $\mathrm{Zu}$ sammenhang zwischen dem Belastungsgrad von Berufen - dargestellt als von den Erwerbstätigen subjektiv wahrgenommene Belastung - und dem Anteil dieser Berufe an den Erwerbsminderungsrenten (z.B. Kistler et al. 2009, S. 13). Der Zugang in Erwerbsminderungsrenten wird aber nicht nur durch die individuelle gesundheitliche Konstitution ermöglicht, sondern auch durch institutionalisierte Verfahren gesteuert, die in ihren Wechselwirkungen bislang nicht detailliert untersucht worden sind. ${ }^{5}$ Der hohe Stellenwert der Institutionalisierung von Erwerbsminderung ist beispielsweise daran sichtbar, dass trotz unverminderter Belastungsexposition die Zugänge in Erwerbsminderungsrenten seit der Reform von 2001 rückläufig sind (vgl. Brussig 2010e).

Arbeitsbelastungen können aber auch zur Aufgabe des bisher ausgeübten Berufes zwingen, selbst wenn damit nicht zwangsläufig die schärfste Konsequenz - die Erwerbsunfähigkeit-verbunden ist. Dies lässt sich anhand der Tätigkeitsverteilung unter den Erwerbstätigen und ihrer Veränderung mit zunehmendem Alter gut nachvollziehen (Brussig 2010a). Gerade in (qualifizierten und unqualifizierten) manuellen Tätigkeiten ist eine vorzeitige Berufsaufgabe zu beobachten. Hierzu zählen neben den sprichwörtlichen Bauberufen auch viele Facharbeiterberufe aus dem industriellen Kern. Umgekehrt nimmt der Anteil unter den dann noch verbliebenen Erwerbstätigen nicht nur bei Hochqualifizierten zu, sondern auch bei Personen in „einfachen Dienstleistungen“. Das bedeutet nicht, dass man in einfachen Dienstleistungen gut alt werden kann. Zu vermuten - wenngleich noch nicht auf der Grundlage individueller Längsschnittdaten nachgewiesen - ist, dass im Alter mehr Personen in „einfache Dienstleistungsberufe“ hineingeströmt als parallel ausgeschieden sind. Selbst wenn den Personen, die aus ihrer angestammten Tätigkeit ausgeschieden sind, eine weitergehende Erwerbstätigkeit z.B. in einfachen Dienstleistungsberufen möglich ist, dürften die langjährig erworbenen Qualifikationen und Kompetenzen in den neuen, aus der Not heraus ergriffenen Tätigkeiten überwiegend nur unterwertig eingesetzt werden.

Ein weiteres seit Langem bestehendes Problem sind die schlechten Wiederbeschäftigungschancen älterer Arbeitsloser, was sich in ihrer langen Arbeitslosigkeitsdauer und in niedrigen Einstellungsraten ausdrückt (Frosch 2007). Mit der Schließung von arbeitslosigkeitsbedingten Frühverrentungsmöglichkeiten gewinnen die Einstellungschancen älterer Arbeitsuchender an Bedeutung. Während ,jüngere Ältere" (50 bis unter 55 Jahre) zwar schon unterdurchschnittliche Einstellungsraten verzeichnen, die aber immerhin dem Konjunkturverlauf folgen, sind die Einstellungsraten der „Älteren“ (60 bis 64 Jahre) nicht nur sehr niedrig, sondern waren zumindest bis 2005 noch dazu von der Konjunktur abgekoppelt und anscheinend auf den „zweiten Arbeitsmarkt“ konzentriert (Brussig 2009).

$\mathrm{Zu}$ den anhaltend schlechten Einstellungschancen passt, dass sich an der schon vielfach konstatierten Ignoranz der betrieblichen Personalpolitik gegenüber dem demografischen Wandel auch in den letzten Jahren anscheinend wenig geändert hat (vgl. Bellmann et al. 2003; Bellmann et al. 2007; Kistler et al. 2010). Die Belegschaften sind zwar deutlich gealtert, doch die Bereitschaft zur Neueinstellung Älterer ist ebenso wie die Weiterbildungsbeteiligung niedrig, und Initiativen zur Verbesserung der Arbeitsbedingungen sind rar. Während Betriebe und Belegschaften unter Zuhilfenahme einer dienstbaren Arbeitsmarktpolitik beim Vorruhestand zusammengewirkt haben, ist eine positive Synergie nach dem arbeitsmarktpolitischen Paradigmenwechsel in Richtung auf längere Erwerbstätigkeit offenbar noch nicht wieder erreicht. Überwiegend nehmen die Betriebe die Alterung ihrer Belegschaften passiv hin.

Darüber hinaus sind neue Probleme in der Alterserwerbstätigkeit entstanden, die zum Teil gerade durch die bereits re- alisierte Heraufsetzung des Rentenalters ausgelöst wurden - und die kaum wahrgenommen werden.

Die Erwerbslosigkeit am Ende des Erwerbslebens verändert ihren Charakter. War Arbeitslosigkeit in den 1990er Jahren noch ein materiell gut gesicherter Weg in die Frühverrentung (Knuth/Kalina 2002), so wird sie zunehmend Bestandteil eines prekären Altersübergangs. Alterserwerbslosigkeit ist in den letzten Jahren zunehmend zu einem Phänomen jenseits des 60. Lebensjahres geworden. In den 1990er Jahren sind Erwerbslose mit dem 60. Lebensjahr sehr oft in eine vorzeitig beziehbare Altersrente gewechselt, was jetzt nicht mehr aufgrund von Arbeitslosigkeit möglich ist. Gleichzeitig hat sich durch die Hartz-Reformen die materielle Absicherung gerade der älteren Arbeitslosen verschlechtert. Die auf Erwerbsintegration gerichtete aktivierende Arbeitsmarktpolitik geht an den Älteren in wesentlichen Teilen nach wie vor vorbei, vor allem bei den 60-Jährigen und Älteren. Bei alldem ist Arbeitslosigkeit einer der stärksten Treiber in die Frühverrentung geblieben, die nun aber wegen versäumter Beitragszahlungen und Rentenabschläge zu spürbar geringeren Renten führt (vgl. Brussig 2010c).

Neuere Untersuchungen zeigen eine verbreitete Instabilität der Beschäftigung in der späten Erwerbsphase (vgl. Brussig 2010b; Zähle et al. 2009) und brechen die noch immer dominante Vorstellung einer hohen Stabilität der beruflichen Position am Ende des Erwerbslebens - sei es in Beschäftigung oder in Arbeitslosigkeit - auf. In Verbindung mit berufsspezifischen Beschäftigungsstrukturen Älterer und dem hohen Stellenwert von geringfügiger, teilzeitiger und zusätzlicher (zum Bezug einer Altersrente oder anderen Transferleistung ausgeübter) Beschäftigung bei Älteren verdichten sich die Indizien, dass ein wesentlicher Teil der Alterserwerbstätigkeit an den Rändern des Arbeitsmarktes stattfindet und die Verlängerung des Erwerbslebens auf der individuellen Ebene vielfach als ein schrittweiser Rückzug erfolgt. Dies würde aber auch bedeuten, dass am Ende des Er-

\footnotetext{
5 Im internationalen Vergleich wird deutlich, dass in Ländern mit "vorbildlich" niedriger Arbeitslosigkeit die Erwerbsminderungsrente (bzw. funktional vergleichbare Leistungen) erheblich stärker in Anspruch genommen wird und stärker als in der Bundesrepublik Deutschland als arbeitsmarktpolitisches Ventil gehandhabt wird (vgl. Erlinghagen/ Knuth 2010).
} 
werbslebens zunehmend Einbußen in der Arbeitsqualität, in den Einkommen und in der sozialen Sicherung hingenommen werden. Vieles an den individuellen Erwerbsverläufen der späten Erwerbsphase ist noch unbekannt, doch die subjektive Dimension - ob es sich beim schrittweisen Rückzug um einen erzwungenen oder individuell angestrebten gleitenden Ausstieg aus dem Arbeitsleben handelt, etwa parallel zum anlaufenden Rentenbezug ist bislang überhaupt nicht erforscht (vgl. dazu auch Abschnitt 5). Geht man aber einmal davon aus, dass Langzeitarbeitslosigkeit am Ende des Erwerbslebens oder ein vorzeitiger Altersrentenbezug von mindestens zwei Jahren einen problematischen Altersübergang anzeigen, dann wies mehr als ein Drittel der Neurentnerinnen und -rentner des Jahres 2009 einen derartigen Altersübergang auf (Abbildung 4).

Die steigenden Risiken beim Altersübergang wirken sich auf das Versorgungsniveau im Alter aus. Trotz des durchschnittlich steigenden Rentenzugangsalters haben sich Rentenabschläge wegen des vorzeitigen Rentenbezugs innerhalb kurzer Zeit verbreitet. Die Altersgrenzen für den abschlagsfreien Rentenzugang sind schneller gestiegen als viele ältere Erwerbspersonen in Form verlängerter Erwerbsbiografien darauf reagieren konnten oder wollten. Gegenwärtig geht etwa die Hälfte der Altersrentnerinnen und -rentner mit Abschlägen in die Rente; in Ostdeutschland sind es bei den Frauen etwa drei Viertel (vgl. Brussig 2010c). Insbesondere Langzeitarbeitslose wechseln zum frühestmöglichen Zeitpunkt in Altersrente, müssen somit massive Abschläge an ihrem Rentenanspruch hinnehmen und erreichen die geringsten durchschnittlichen Rentenzahlbeträge (ebd.). Generell wird mit einer wachsenden Altersarmut gerechnet (vgl. Steiner/Geyer 2010; Wübbeke 2007), wobei offen ist, welche Rolle dabei die prekären Altersübergänge und welche Rolle unstetige Erwerbsverläufe insgesamt spielen.

\section{Zusammenfassung und Ausblick}

Bereits die zurückliegenden 15 Jahre waren durch Arbeitsmarkt- und Rentenreformen geprägt, die in ihrer Reichweite kaum hinter der „Rente mit 67“ zurückstehen, son-

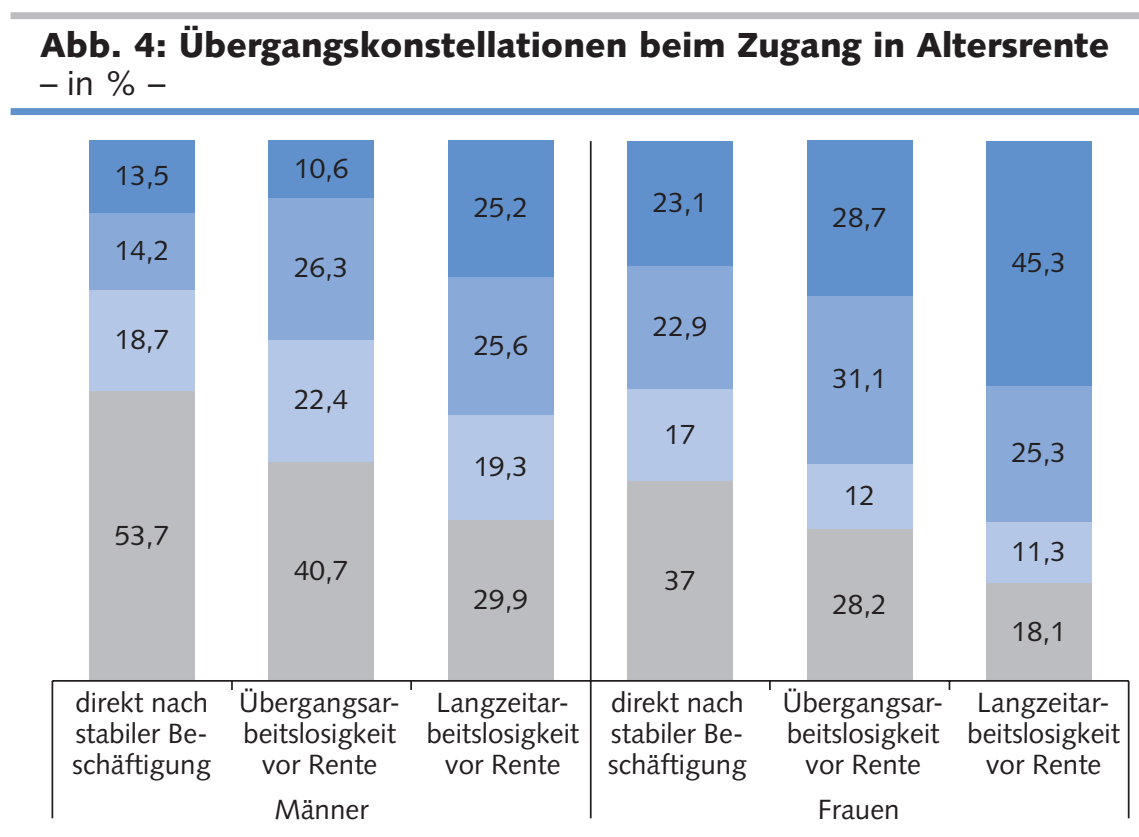

Monate des vorzeitigen Rentenbezugs:

$\square$ keine Abschläge $\square 1$ bis unter 24 Monate $\square 24$ bis unter 60 Monate $\square 0$ Monate

Quelle: Brussig 2010c auf Grundlage des FDZ-RV SUF RTZNO7XVSBB

WSI MitTEILUNGeN

dern sie eher übertreffen. Es sind erhebliche Verhaltensänderungen zu verzeichnen, die sich durchaus in der beabsichtigten Richtung in einem Ausmaß bewegen, das zunächst kaum für möglich gehalten wurde. Gleichwohl hat ein erheblicher Teil der Beschäftigten Probleme, bis zum Erreichen des 65. Lebensjahres erwerbstätig zu sein, und ein weiterer Teil schafft dies nur in geringfügiger und teilzeitiger Beschäftigung.

Die Probleme, die Erwerbsphasen auszudehnen, lassen sich zu zwei Komplexen zusammenfassen: Der eine Problemkreis betrifft die fehlende individuelle Leistungsfähigkeit im Alter - wesentlich bedingt durch Gesundheitsprobleme -, die zu vorzeitiger Berufsaufgabe und dem Abdrängen in randständige Jobs oder Arbeitslosigkeit (und trotzdem nur vergleichsweise selten in eine Erwerbsminderungsrente) führt. Der zweite Problemkreis betrifft die fehlende betriebliche Nachfrage, ältere Mitarbeiter neu einzustellen, was die Integration älterer Arbeitsloser erschwert und ebenfalls instabile Erwerbsverläufe am Ende des Erwerbslebens provoziert.

Über die vielfältigen Forderungen an Gesetzgeber, Tarifpartner, Betriebe, Arbeitnehmer, aber beispielsweise auch gegenüber der öffentlichen Arbeitsvermittlung, sollte nicht aus dem Blick geraten, dass die nun einsetzende Anhebung der Altersgrenze über das 65. Lebensjahr hinaus auch neue Anforderungen an die Beobachtung und Erklärung der Alterserwerbstätigkeit und des Übergangs von Erwerbstätigkeit in Rente stellt.

Über die bereits verfügbaren Informationen zur Alterserwerbstätigkeit, Beschäftigungslosigkeit im Alter und zum Zugang in die Altersrente hinaus betrifft dies die Qualität der Beschäftigung, die am Ende des Erwerbslebens ausgeübt wird. Mit einer bloßen Differenzierung in versicherungspflichtige und atypische Beschäftigung in ihren verschiedenen Formen wird es nicht getan sein. Wissensrelevant sind auch Arbeitszeiten und Einkommensverläufe sowie die Wahrnehmung von Arbeitsbelastungen. Von zunehmendem Interesse wird die Überlagerung von Erwerbstätigkeit und Rentenbezug sowohl vor Erreichen der Regelaltersgrenze - wofür es Zuverdienstgrenzen gibt - als auch nach dem Erreichen der Altersgrenze sein. Nahezu völlig unbekannt sind Tätigkeitswechsel in der späten Erwerbsphase: Wie realistisch ist die Vorstellung, am Ende des Erwerbslebens aus hoch belastenden Tätigkeiten in weniger belastende Tätigkeiten zu wechseln, ohne dass dabei die individuellen Kompetenzen und Erfahrungen verfallen?

Der subjektiven Wahrnehmung des Alterübergangs wurde schon in den Analysen der 1980er und 90er Jahre zum Vorruhestand relativ wenig Aufmerksamkeit 
geschenkt, in denen oftmals nur davon ausgegangen wurde, dass der Vorruhestand von den Arbeitnehmerinnen und Arbeitnehmern ebenso angestrebt wurde wie von den Betrieben. Doch in den aktuellen Untersuchungen fehlt die subjektive Dimension nahezu vollständig. Die vorhandenen Befragungen zum geplanten Renteneintrittsalter oder zur Vorstellung künftiger Erwerbstätigkeit im Rentenalter sind zwar aufschlussreich. Doch sicherlich von größerem Interesse ist ein Einblick in die subjektiven Abwägungen von Handlungsalternativen, etwa zur Inkaufnahme von Rentenabschlägen, zur Fortführung, Beendigung und Wiederaufnahme von Erwerbstätigkeit oder bei Tätigkeitswechseln. Möglicherweise klärt sich dann auch der offenkundige Widerspruch zwischen der langjährigen Konstanz der wahrgenommenen Arbeitsbelastungen einerseits und der signifikanten Verlängerung individueller Erwerbsphasen andererseits, wie er sich aus den aktuellen Studien ergibt.

Die angesprochenen Aspekte zu den Strukturen der Alterserwerbstätigkeit und von Altersübergängen sowie zu ihrer subjektiven Dimension sind auch deshalb unbekannt, weil erstens die zugrunde liegenden Forschungsfragen erst mit der Anhebung der Altersgrenzen relevant werden. Zweitens lagen die dafür erforderlichen Daten lange nicht vor. Dies hat sich für die strukturellen Aspekte durch die Forschungsdatenzentren der Deutschen Rentenversicherung und des Instituts für Arbeitsmarkt- und Berufsforschung (IAB) wesentlich verbessert, sodass in den kommenden Jahren substanzielle Erkenntnisgewinne zur Alterserwerbstätigkeit und zu Altersübergängen zu erwarten sind.

\section{LITERATUR}

Bellmann, L./Kistler, E./Wahse, J. (2003): Betriebliche Sicht- und Verhaltensweisen gegenüber älteren Arbeitnehmern, in: Aus Politik und Zeitgeschichte 53 (20), S. 26-34

Bellmann, L./Kistler, E./Wahse, J. (2007): Demographischer Wandel: Betriebe müssen sich auf alternde Belegschaften einstellen, hrsg. vom Institut für Arbeitsmarkt und Berufsforschung, IAB-Kurzbericht (21), Nürnberg

Böckler, M. (2010): Fachkräftemangel im Ruhrgebiet. Perspektiven des Arbeitskräftepotenzials in der Konkurrenz der Regionen, hrsg. vom Institut Arbeit und Qualifikation, IAQ-Report (05), Duisburg

Börsch-Supan, A. (2010): Generationengerechtigkeit in der Alterssicherung, in: Deutsche Rentenversicherung Bund (Hrsg.): Gerechtigkeitskonzepte und Verteilungsströme in der gesetzlichen Alterssicherung, Jahrestagung 2010 des Forschungsnetzwerkes Alterssicherung (FNA) am 28. und 29. Januar in Berlin, DRV-Schriften (89), S. 47-52

Brussig, M. (2009): Neueinstellungen von Älteren: Keine Ausnahme, aber auch noch keine Normalität. Anhaltende Altersungleichheit bei Neueinstellungen trotz zunehmender Beschäftigungsquoten Älterer, Altersübergangs-Report (01), Düsseldorf/Duisburg-Essen

Brussig, M. (2010a): Erwerbstätigkeit im Alter hängt vom Beruf ab. Ausdifferenzierung der Erwerbschancen vor allem nach dem 60. Lebensjahr, in einigen Berufen aber schon früher, Altersübergangs-Report (05), Düsseldorf/Duisburg-Essen

Brussig, M. (2010b): Erwerbsverläufe vor dem Rentenbeginn. Eine Analyse mit der VVL 2004, in: Himmelreicher, R. K. (Hrsg.): FDZ-RVDaten zur Rehabilitation, über Versicherte und Rentner, 6. Workshop des FDZ-RV, DRV-Schriften 55/2009, S. 231-249

Brussig, M. (2010c): Fast die Hälfte aller neuen Altersrenten mit Abschlägen - Quote weiterhin steigend. Probleme mit dem Anstieg der Altersgrenzen vor allem bei Arbeitslosen, aber auch bei Erwerbstätigen, Altersübergangs-Report (01), Düsseldorf/Duisburg-Essen
Brussig, M. (2010d): Höhere Alterserwerbsbeteiligung durch längere Erwerbsphasen. In jüngeren Kohorten sind mehr Menschen länger erwerbstätig als in älteren Kohorten, Altersübergangs-Report (04), Düsseldorf/Duisburg-Essen

Brussig, M. (2010e): Künftig mehr Zugänge in Altersrenten absehbar - Gegenwärtig kein Ausweichen in die Erwerbsminderungsrente zu beobachten, Altersübergangs-Report (02), Düsseldorf/Duisburg-Essen Bundesministerium für Arbeit und Soziales (BMAS) (2010): Aufbruch in die altersgerechte Arbeitswelt, Bericht der Bundesregierung gemäß $\S 154$ Abs. 4 Sechstes Buch Sozialgesetzbuch zur Anhebung der Regelaltersgrenze auf 67 Jahre, Berlin

Büttner, R. (2005): Die Wirkungsweise der Rentenreformen auf den Altersübergang, Altersübergangs-Report (01), Gelsenkirchen

Dobritz, J./Micheel, F. (2010): Weiterbeschäftigung im Rentenalter - Potenziale, Einstellungen und Bedingungen, in: Bevölkerungsforschung, Mitteilungen aus dem Bundesinstitut für Bevölkerungsforschung 31 (3), S. 1-7 Ebert, A./Fuchs, T./Kistler, E. (2006): Arbeiten bis 65 oder gar bis 67 ? - Die Voraussetzungen fehlen, in: WSI-Mitteilungen 59 (9), S. 492-499

Engstler, H. (2004): Geplantes und realisiertes Austrittsalter aus dem Erwerbsleben. Ergebnisse des Alterssurveys 1996 und 2002, Deutsches Zentrum für Altersfragen, DZA-Diskussionspapier (41), Berlin

Erlinghagen, M./Knuth, M. (2010): Unemployment as an institutional construct? Structural differences in non-employment between selected European countries and the United States, in: Journal of Social Policy 39 (1), S. 71-94

Frosch, K. (2007): Zu alt für einen neuen Job. Altersspezifische Wiederbeschäftigungschancen nach Verlust des Arbeitsplatzes, in: Wirtschaftspolitische Blätter 54 (4), S. 641-656

Fuchs, J. (2006): Rente mit 67: Neue Herausforderungen für die Beschäftigungspolitik, IAB-Kurzbericht (16), Nürnberg 
Fuchs, J./Zika, G. (2010): Arbeitsmarktbilanz bis 2025: Demografie gibt die Richtung vor, IAB-Kurzbericht (12), Nürnberg

Guggemos, P./Huber, A./Kistler, E. (2007): Wohin am Arbeitsmarkt mit der künftig viel größeren Zahl älterer Erwerbspersonen, in: Stecker, Ch./Putzing, M./Hinz, P./Kaufmann, I. (Hrsg.): Smart Region. Projektergebnisse zum alternsgerechten Arbeiten in innovativen Regionen, DRV-Schriften (70), S. 45-64

Kistler, E./Ebert, A./Trischler, F./Bäcker, G. (2009): Rente mit 67 - die Voraussetzungen stimmen nicht! Erster Monitoring-Bericht des Netzwerks für eine gerechte Rente, Sozialverband Deutschland e.V. Berlin Kistler, E./Trischler, F./Bäcker, G. (2010): Rente mit 67? Zu wenig Arbeitsplätze und zu wenig gute Arbeit für ein Arbeiten bis 67. Vierter Monitoring-Bericht des Netzwerks für eine gerechte Rente, Deutscher Paritätischer Wohlfahrtsverband, Berlin

Knuth, M./Kalina, Th. (2002): Early exit from the labour force between exclusion and privilege: Unemployment as a transition from employment to retirement in West Germany, in: European Societies 4 (4),

S. $393-418$
Lutz, B. (2010): Fachkräftemangel in Ostdeutschland - Konsequenzen für Beschäftigung und Interessenvertretung, unter Mitarbeit von Grünert, H./Ketzmerick, Th./Wiekert, I., Otto-Brenner-Stiftung, OBSArbeitsheft (65), Frankfurt am Main

Steiner, V./Geyer, J. (2010): Künftige Altersrenten in Deutschland: relative Stabilität im Westen, starker Rückgang im Osten, in: DIWWochenbericht 82 (11), S. 2-11

Trischler, F./Kistler, E. (2010): Gute Erwerbsbiographien. Arbeitsbedingungen und Erwerbsverlauf, Arbeitspapier (2), Stadtbergen

Wübbeke, Ch. (2007): Ältere Bezieher von Arbeitslosengeld II: Einmal arm, immer arm, IAB-Kurzbericht (14), Nürnberg

Zähle, T./Möhring, K./Krause, P. (2009): Erwerbsverläufe beim Übergang in den Ruhestand, in: WSI-Mitteilungen 62 (11), S. 586-595 